\title{
Continuants, run lengths, and Barry's modified Pascal triangle
}

\author{
Lukas Spiegelhofer* \\ Institut für diskrete Mathematik und Geometrie \\ Technische Universität Wien \\ Wiedner Hauptstrasse 8-10, 1040 Wien, Austria \\ lukas.spiegelhofer@tuwien.ac.at
}

\author{
Jeffrey Shallit ${ }^{\dagger}$ \\ School of Computer Science \\ University of Waterloo \\ Waterloo, ON N2L 3G1, Canada \\ shallit@uwaterloo.ca
}

Submitted: Oct 17, 2017; Accepted: Aug 20, 2018; Published: Mar 8, 2019

(C) The authors. Released under the CC BY license (International 4.0).

\begin{abstract}
We show that the $n$ 'th diagonal sum of Barry's modified Pascal triangle can be described as the continuant of the run lengths of the binary representation of $n$. In other words, Stern's diatomic sequence appears in these diagonal sums. We also obtain an explicit description for the row sums.
\end{abstract}

Mathematics Subject Classifications: Primary: 05A10; Secondary: 05A19, $11 \mathrm{~A} 55,11 \mathrm{~A} 63$

\section{Introduction}

In 2006 in the On-Line Encyclopedia of Integer Sequences (OEIS) [9], sequence A119326, Paul Barry introduced a modified Pascal triangle, defined for integers $k$ and $n$ such that $0 \leqslant k \leqslant n$, as follows:

$$
T(n, k)=\sum_{\substack{0 \leqslant j \leqslant n-k \\
2 \mid j}}\left(\begin{array}{c}
k \\
j
\end{array}\right)\left(\begin{array}{c}
n-k \\
j
\end{array}\right) .
$$

The first few rows of this triangle are as follows:

${ }^{*}$ The author acknowledges support by project F5502-N26 (FWF), which is a part of the Special Research Program "Quasi Monte Carlo Methods: Theory and Applications". Moreover, we thank the Erwin Schrödinger Institute for Mathematics and Physics for providing the opportunity to carry out research work during his visit for the programme "Tractability of High Dimensional Problems and Discrepancy"

†The author is supported by a grant from NSERC

Key words and phrases. modified Pascal triangle, continued fraction, continuant, Stern's diatomic sequence 


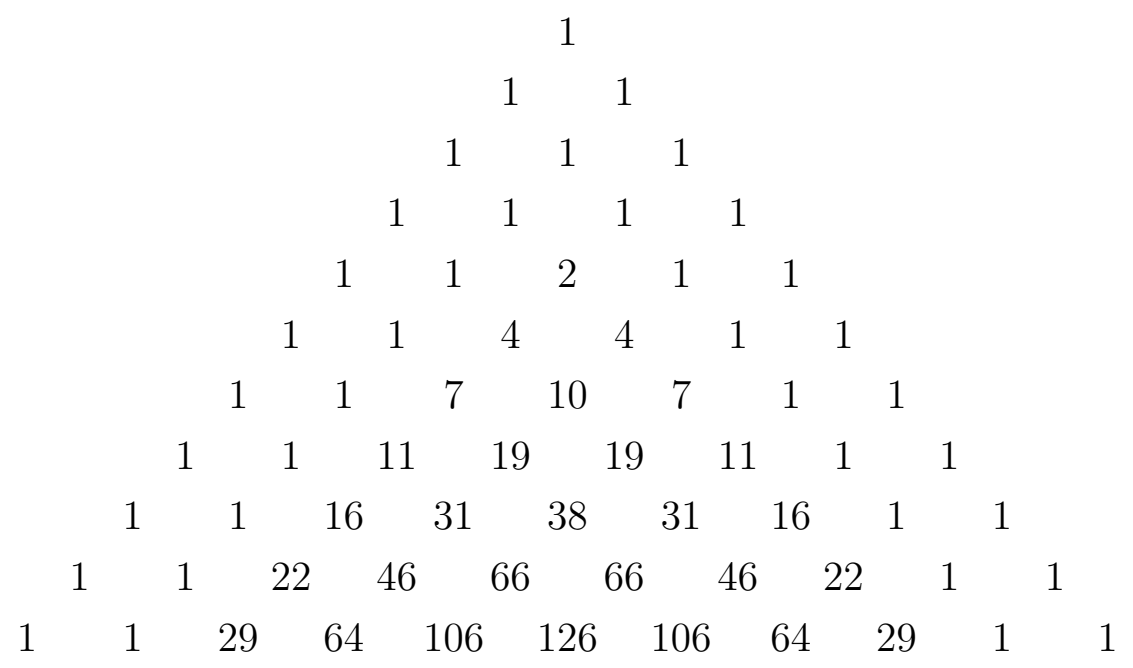

Similarly, one can consider $T(n, k) \bmod 2$, whose terms are given by sequence A114213:

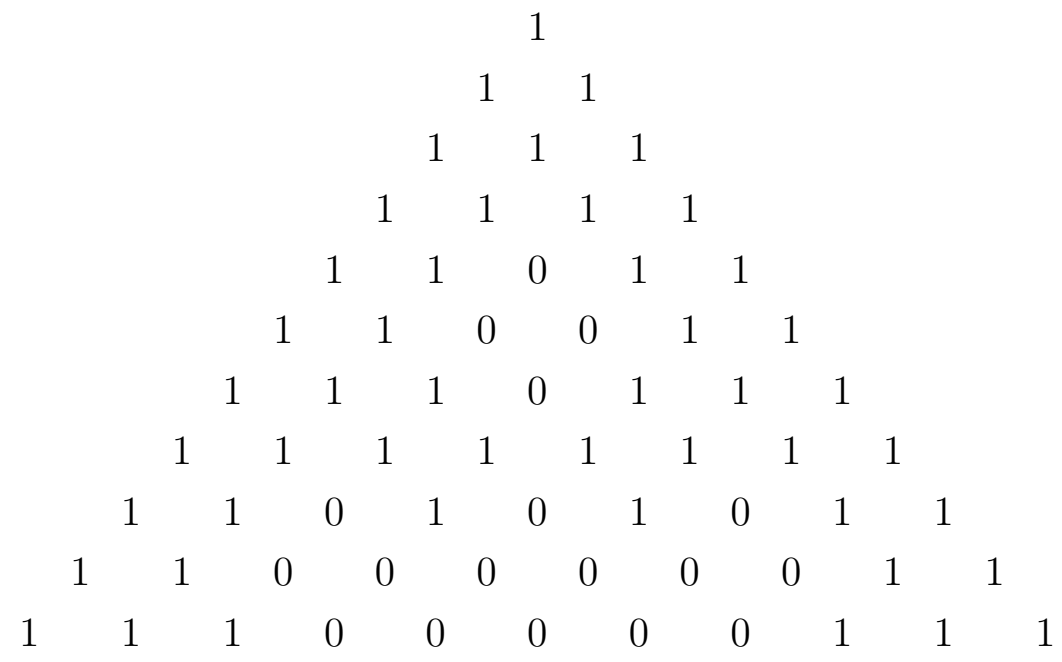

Sequences A114212 and A114214, respectively, are the row sums and diagonal sums of this latter triangle. We denote them by $r(n)$ and $d(n)$, respectively:

$$
\begin{aligned}
r(n) & =\sum_{k=0}^{n}(T(n, k) \bmod 2) \\
d(n) & =\sum_{k=0}^{\lfloor n / 2\rfloor}(T(n-k, k) \bmod 2) .
\end{aligned}
$$

In May 2016, the first author observed, empirically, a connection between $d(n)$ and the binary representation of $n$. In this note we prove this connection, and also prove a formula for $r(n)$. The connection involves Stern's "diatomic sequence" $s(n)$, defined by $s(0)=0, s(1)=1, s(2 n)=s(n)$, and $s(2 n+1)=s(n)+s(n+1)$; see [10]. 


\section{The diagonal sums}

Let the binary representation of $n$ be denoted by $\sum_{i=0}^{j} \varepsilon_{i}(n) 2^{i}$. We show that the diagonal sum $d(n)$ can be expressed in terms of this representation. Given a string $s$ of 0 's and 1 's, we consider its run lengths: the lengths of maximal blocks of consecutive identical elements. For example, if $s=111000011111$, then the run lengths of $s$ are $(3,4,5)$.

If $m$ is a sequence of positive integers, we may associate an integer with it via the continued fraction expansion: if $m=\left(m_{0}, \ldots, m_{k}\right)$, we say that the continuant of $m$ is the numerator of the continued fraction $\left[m_{0} ; m_{1}, \ldots, m_{k}\right]$ (see $[4$, Ch. 34, §4]).

Theorem 1. Let $n \geqslant 0$ be an integer and let $m$ be the sequence of run lengths of the binary representation of $n$. Then $d(n)$ equals the continuant of $m$.

We will use Lucas' famous congruence for binomial coefficients [8, p. 230]: if $p$ is a prime number and $n=\left(n_{\nu} \cdots n_{0}\right)_{p}$ and $k=\left(k_{\nu} \cdots k_{0}\right)_{p}$, then

$$
\left(\begin{array}{l}
n \\
k
\end{array}\right) \equiv\left(\begin{array}{l}
n_{\nu} \\
k_{\nu}
\end{array}\right) \cdots\left(\begin{array}{l}
n_{0} \\
k_{0}
\end{array}\right) \quad(\bmod p) .
$$

This implies that $\left(\begin{array}{l}n \\ k\end{array}\right)$ is not divisible by $p$ if and only if $k_{i} \leqslant n_{i}$ for all $i$.

We prove the following statement, which reduces the problem to divisibility by 2 of binomial coefficients. We will derive Theorem 1 from it in a moment.

Proposition 2. Let $n$ and $k$ be nonnegative integers such that $k \leqslant n$. If $2 \mid n+k$, then $T(n, k) \equiv\left(\begin{array}{l}n \\ k\end{array}\right)(\bmod 2)$. Otherwise, $T(n, k) \equiv\left(\begin{array}{c}n-1 \\ k\end{array}\right)(\bmod 2)$.

Proof. We prove the first statement. By replacing $n$ with $n+k$ we get the equivalent assertion that if $2 \mid n$ or $2 \mid k$, then

$$
\sum_{\substack{0 \leqslant j \leqslant n \\
2 \mid j}}\left(\begin{array}{l}
n \\
j
\end{array}\right)\left(\begin{array}{l}
k \\
j
\end{array}\right) \equiv\left(\begin{array}{c}
n+k \\
k
\end{array}\right) \quad(\bmod 2) .
$$

By Lucas' congruence the left-hand side is congruent to

$$
\sum_{j=0}^{n}\left(\begin{array}{l}
n \\
j
\end{array}\right)\left(\begin{array}{l}
k \\
j
\end{array}\right) \equiv \sum_{j=0}^{n}\left(\begin{array}{c}
n \wedge k \\
j
\end{array}\right) \equiv 2^{s_{2}(n \wedge k)} \quad(\bmod 2),
$$

where $n \wedge k$ is the integer whose binary digits satisfy $\varepsilon_{i}(n \wedge k)=\min \left(\varepsilon_{i}(n), \varepsilon_{i}(k)\right)$. This expression is odd if and only if $s_{2}(n \wedge k)=0$, which is the case if and only if the binary representations of $n$ and $k$ are disjoint. To handle the right-hand side of Eq. (1), we note that $\left(\begin{array}{c}n+k \\ k\end{array}\right)$ is odd if $n \wedge k=1$. On the other hand, if the binary representations of $n$ and $k$ are not disjoint, then the condition $\varepsilon_{i}(k) \leqslant \varepsilon_{i}(n+k)$ is violated for $i=\min \left\{j: \varepsilon_{j}(n)=\right.$ $\left.1, \varepsilon_{j}(k)=1\right\}$; therefore $\left(\begin{array}{c}n+k \\ k\end{array}\right)$ is even. This proves the first assertion.

For the second assertion, we use Lucas' congruence again: for $2 \mid j$ and $2 \mid m$ we have $\left(\begin{array}{c}m \\ j\end{array}\right) \equiv\left(\begin{array}{c}m+1 \\ j\end{array}\right)(\bmod 2)$. Since $2 \nmid n-k$, we obtain $\left(\begin{array}{c}n-k \\ j\end{array}\right) \equiv\left(\begin{array}{c}n-1-k \\ j\end{array}\right)(\bmod 2)$. Moreover, by $2 \nmid n-k$ the ranges of summation in $T(n, k)$ and $T(n-1, k)$ are the same. 
From this proposition we obtain in particular the identity

$$
d(2 n)=d(2 n+1)
$$

Carlitz [3] proved that Stern's diatomic sequence $s(n)$ satisfies $s(n+1)=\sum_{k=0}^{\lfloor n / 2\rfloor}\left(\left(\begin{array}{c}n-k \\ k\end{array}\right) \bmod 2\right)$. By Proposition 2 and Eq. (2) we therefore have

$$
d(2 n)=d(2 n+1)=s(2 n+1) .
$$

Of course, by the recurrence for $s$ this can be written as

$$
d(n)=s(\lfloor n / 2\rfloor)+s(\lfloor n / 2\rfloor+1) .
$$

It is well-known $[6,7]$ that if $m=\left(m_{0}, \ldots, m_{k}\right)$ is the sequence of run-lengths of the binary representation of $n$ and $n$ is odd, then $s(n)$ is the continuant of $m$. Therefore $d(n)$ is the continuant of $m$. In order to complete the proof of the conjecture, we have to show that the same is true for even $n$. By Eq. (3) it is sufficient to prove the following lemma.

Lemma 3. If $n$ is even, then the continuant of the sequence of run-lengths of the binary representation of $n$ is equal to the continuant corresponding to $n+1$.

Proof. Let $n=1^{m_{0}} 0^{m_{1}} \cdots 1^{m_{k-1}} 0^{m_{k}}$. We distinguish between two cases. If $m_{k}=1$, then $n+1=\left(1^{m_{0}} 0^{m_{1}} \cdots 0^{m_{k-2}} 1^{m_{k-1}+1}\right)$ and the statement follows from the identity $\left[m_{0} ; m_{1}, \ldots, m_{k-1}, 1\right]=\left[m_{0} ; m_{1}, \ldots, m_{k-1}+1\right]$.

If $m_{k} \geqslant 2$, then $n+1=\left(1^{m_{0}} 0^{m_{1}} \cdots 0^{m_{k-2}} 1^{m_{k-1}} 0^{m_{k}-1} 1\right)$ and the statement follows from $\left[m_{0} ; m_{1}, \ldots, m_{k}\right]=\left[m_{0} ; m_{1}, \ldots, m_{k-1}, m_{k}-1,1\right]$.

Remark. The sequence $(d(n))_{n \geqslant 0}$ is a 2-regular sequence [1], as it satisfies the equalities

$$
\begin{aligned}
d(2 n+1) & =d(2 n) \\
d(4 n+2) & =3 d(2 n)-d(4 n) \\
d(8 n) & =-d(2 n)+2 d(4 n) \\
d(8 n+4) & =4 d(2 n)-d(4 n) .
\end{aligned}
$$

\section{The row sums}

In order to state our result, we define the sum-of-digits function $s_{2}$ in base 2: this function returns the number of $1 \mathrm{~s}$ in the binary expansion of a nonnegative integer $n$. By Lucas' congruence, it follows that the number of odd binomial coefficients $\left(\begin{array}{l}n \\ k\end{array}\right)$ equals $2^{s_{2}(n)}[5]$.

We will prove an analogous result for $r(n)$.

\section{Theorem 4.}

$$
r(n)= \begin{cases}2^{s_{2}(n)}, & \text { if } n \text { odd } \\ 2^{s_{2}(n)}+2^{s_{2}(n-2)}, & \text { if } n \text { even }\end{cases}
$$


Again, this expression can be written in a unified way:

$$
r(n)=2^{s_{2}(\lfloor n / 2\rfloor)}+2^{s_{2}(\lfloor(n-1) / 2\rfloor)} .
$$

A similar characterization was stated, without proof or attribution, in the notes to A114212 of the OEIS.

Proof. From Proposition 2 we get, for integers $n \geqslant k \geqslant 0$, that

$$
\begin{aligned}
T(2 n, 2 k) & \equiv T(2 n+1,2 k) \equiv T(2 n+1,2 k+1) \equiv\left(\begin{array}{l}
n \\
k
\end{array}\right)(\bmod 2) ; \\
T(2 n, 2 k+1) & \equiv\left(\begin{array}{c}
n-1 \\
k
\end{array}\right)(\bmod 2) .
\end{aligned}
$$

Then

$$
\begin{aligned}
r(2 m) & =\sum_{k=0}^{2 m}(T(2 m, k) \bmod 2) \\
& =\sum_{k=0}^{m}(T(2 m, 2 k) \bmod 2)+\sum_{k=0}^{m-1}(T(2 m, 2 k+1) \bmod 2) \\
& =\sum_{k=0}^{m}\left(\left(\begin{array}{c}
m \\
k
\end{array}\right) \bmod 2\right)+\sum_{k=0}^{m-1}\left(\left(\begin{array}{c}
m-1 \\
k
\end{array}\right) \bmod 2\right) \\
& =2^{s_{2}(m)}+2^{s_{2}(m-1)} \\
& =2^{s_{2}(2 m)}+2^{s_{2}(2 m-2)} .
\end{aligned}
$$

Similarly,

$$
\begin{aligned}
r(2 m+1) & =\sum_{k=0}^{2 m+1}(T(2 m+1, k) \bmod 2) \\
& =\sum_{k=0}^{m}(T(2 m+1,2 k) \bmod 2)+\sum_{k=0}^{m}(T(2 m+1,2 k+1) \bmod 2) \\
& =\sum_{k=0}^{m}\left(\left(\begin{array}{c}
m \\
k
\end{array}\right) \bmod 2\right)+\sum_{k=0}^{m}\left(\left(\begin{array}{c}
m \\
k
\end{array}\right) \bmod 2\right) \\
& =2^{s_{2}(m)}+2^{s_{2}(m)} \\
& =2^{s_{2}(2 m+1)}
\end{aligned}
$$

\section{Acknowledgements}

We thank Paul Barry for providing more details about the motivation for his sum [2]. 


\section{References}

[1] J.-P. Allouche And J. O. Shallit, The ring of k-regular sequences, Theoret. Comput. Sci., 98 (1992), pp. 163-197.

[2] P. BARrY, Notes on A114213. Unpublished manuscript, October 31, 2017.

[3] L. Carlitz, Single variable Bell polynomials, Collect. Math., 14 (1962), pp. 13-25.

[4] G. Chrystal, Algebra, vol. 2, Adam and Charles Black, 1900.

[5] J. W. L. GLAisher, On the residue of a binomial-theorem coefficient with respect to a prime modulus, Quarterly J. Pure Appl. Math., 30 (1899), pp. 150-156.

[6] D. H. Lehmer, On Stern's diatomic series, Amer. Math. Monthly, 36 (1929), pp. 5967.

[7] D. A. Lind, An extension of Stern's diatomic series, Duke Math. J., 36 (1969), pp. 55-60.

[8] É. LuCAS, Théorie des fonctions numériques simplement périodiques, Amer. J. Math., 1 (1878), pp. 197-240.

[9] N. J. A. Sloane et AL., The On-Line Encyclopedia of Integer Sequences, 2019. Published electronically at https://oeis.org.

[10] M. A. Stern, Über eine zahlentheoretische Funktion, J. Reine Angew. Math., 55 (1858), pp. 193-220. 